\title{
Role and training of nurses working in departments of genitourinary medicine in England and Wales
}

\author{
2. Training and future role
}

\author{
JACQUELINE S ROGERS AND MICHAEL W ADLER \\ From the Academic Department of Genitourinary Medicine, Middlesex Hospital Medical School, \\ London
}

\section{Introduction}

Our previous paper described the background and methods used in an inquiry into the role of nurses working in departments of genitourinary medicine (GUM). ${ }^{1}$ This paper is concerned particularly with training, teaching, and the future role of these nurses.

\section{Training and teaching}

One of the aims of the study was to indicate educational programmes that could be developed to train nurses in the specialty, so it was necessary to identify the training that was being given at the time of the interview.

\section{TEACHING STUDENT NURSES}

The General Nursing Council lays down no specific guidelines concerning the time or content of nurse teaching in sexually transmitted disease (STD). It only requires that male nurses spend a short time working in the clinic during their general training period.

Doctors were asked if they taught student or pupil nurses during their general training in the hospital in which the interview was conducted. Of the 69 doctors interviewed, 51 (74\%) taught whereas $18(26 \%)$ said they did not. Of the 18 who did not teach, nine said that another doctor taught in the school of nursing, seven said that the nurse tutors gave all the teaching for that specialty, and two said that they had never been asked.

Doctors who did teach student or pupil nurses were asked how many hours a month were spent in the school of nursing, and what topics were usually covered. The mean time given to teaching was one hour (range 25 minutes to six hours) a month. Doctors generally felt that, because of the limited amount of time alotted to the specialty, only certain aspects could be highlighted. Teaching tended to be in lecture form,

Address for reprints: Professor M W Adler, Academic Department of Genitourinary Medicine, Middlesex Hospital Medical School, London W1

Accepted for publication 6 August 1986 though some doctors actively encouraged the students to visit the clinic when this was feasible. Some doctors attempted to cover all the possible infections briefly, some concentrated on a few most often seen in clinics, whereas others spoke very briefly of the diseases and concentrated on the availability of clinics, contact tracing, and possible prevention of disease.

\section{TEACHING NURSING STAFF IN CLINICS \\ The teachers}

The doctors were asked about teaching the nurses who work in the clinic. Sixty two (90\%) of those interviewed taught the nurses in their department. In clinics where other medical or senior nursing staff were employed, these staff also contributed towards the teaching. The method most often used (by 52 (75\%) of doctors) was occasional teaching using specific patients. Twenty three (37\%) doctors taught by observation, which included being present at the consultation and teaching at the examination. Only $13(21 \%)$ doctors used more formal teaching methods such as lectures, seminars, or discussion groups.

Doctors found it difficult to assess the amount of time devoted to teaching in the clinics because of its informal structure. The mean hours a month were, however, calculated as being 1.9 (range 0.25 to 7 ). This time was often snatched between patient consultations or in a coffee break. Seven doctors were unable to teach their clinic nurses. The reasons they gave for this were: not enough time, space, or staff; nurses had been in post too long to be taught; and staff changed too often to make it worthwhile.

\section{The taught}

Of the 118 nurses interviewed, 114 (97\%) said they had been given some degree of training by a staff member, whereas three nurses $(3 \%)$ said they had started working in the clinic with no guidance from anybody. The nurses were asked who had given them their training and, as one would expect in the smaller clinics, $95(81 \%)$ replied they had been taught by the doctor and $76(64 \%)$ by a senior nurse. Only five nurses (4\%) had had any training from a nurse tutor 
while working in the clinic, and $18(15 \%)$ had been taught by technicians, junior nursing staff, or clerical staff.

The content of the teaching varied. The traditional role of the nurse in preparing the patient and giving treatment were given highest teaching priority (104 (88\%) nurses were taught about this) followed by the less traditional role of taking diagnostic tests $(102$, $86 \%$ ) and examining the genitalia $(99,84 \%)$. Setting up the clinic (preparing the instruments, cultures, and microscopes and in some cases finding patients' notes) were taught to $94(80 \%)$ of the nurses. Venepuncture and microscopy, both specialist skills, were taught to $93(79 \%)$ and $89(75 \%)$ nurses, respectively. Skills that were taught to fewer nurses were special procedures for female and male patients (to 56 (47\%) and 45 (38\%) nurses respectively) (which included cytology, bimanual examination, proctoscopy, and urethral dilatation) and contact tracing (to 45 (38\%) nurses).

The nurses were asked if they had been given any theoretical teaching. Ninety five $(80 \%)$ nurses had been taught which tests were taken, why they were taken, the interpretation of the results, and treatment given, and 93 (79\%) the clinical manifestations of the diseases. The ethical and medicolegal aspects of the specialty were thought to be important by doctors and nurses, which is reflected in the number of nurses $(84$, $71 \%$ ) who had had teaching on this subject. Eighty one (69\%) nurses had been given some teaching in microbiology, though most admitted that their knowledge was very limited. The subject of serology had been taught to $76(64 \%)$, and $68(58 \%)$ nurses had been taught something of the theory of contact tracing and counselling patients. The subject given the least attention was the anatomy and physiology of the urinary and reproductive systems.

The nurses were further asked how adequate they felt the teaching had been. Three of the 118 nurses did not give answers to this, but of those who did, 65 (56\%) were satisfied but $48(42 \%)$ felt that they needed more. Particular inadequacies were strongly felt by some, whereas other nurses were vague, only being able to say that they knew too little of the subject and felt a need for teaching in every aspect. The nurses felt that theoretical teaching was the most neglected area (table I).

Seventy three $(62 \%)$ of the original 118 nurses interviewed were responsible for teaching other learners visiting the clinic. The level of tuition was not recorded, but in many clinics visiting doctors or medical students, student nurses or pupil midwives, or nurses on other relevant courses would have been taught. Some clinics had occasional visits from groups, such as prison officers, students, and probation officers.

Opportunities for self education in the specialty
TABLE I No (\%) of 115 senior nurses who thought that teaching subjects listed were neglected

\begin{tabular}{lr}
\hline Neglected subjects & No $(\%) *$ \\
\hline Theoretical and practical teaching & $31(27 \cdot 0)$ \\
Technical skills, such as microscopy & $10(8 \cdot 7)$ \\
Contact tracing & $9(7 \cdot 8)$ \\
Importance of STS and laboratory work & $7(6 \cdot 1)$ \\
Counselling techniques & $6(5 \cdot 2)$ \\
Training before starting jobs & $4(3 \cdot 5)$ \\
$\quad$ legal and ethical aspects) & $1(0 \cdot 9)$ \\
Anatomy and physiology & 1
\end{tabular}

* 16 nurses gave more than one subject.

STS $=$ serological tests for syphilis.

were few. Though 95 (81\%) nurses said they had access to current relevant books and journals, these often proved to be in the nursing school library or locked in the doctor's desk drawer. Little money seemed to be spent on books and journals for the clinics. Few nurses (only $25 \%$ ) had the opportunity to attend meetings concerned with the specialty either in or outside their own hospital. Few clinics were large enough to warrant enough inpatient beds to hold ward rounds, and only $5 \%$ of the sample (six nurses) attended ward rounds.

\section{ESTABLISHED TRAINING FOR NURSES}

The English National Board (ENB) has approved a six month course for state registered and state enrolled nurses (course 275), which is designed to "prepare (them) to fulfil a specified role in the clinic." The course is run at two centres in England, and four to six students are accepted on each six month course. The courses have been running for 10 years, and so far 214 nurses have attended. In addition to this long course, the ENB has also approved a short five day updating course (course 932) for nurses with a minimum of three years' experience in the specialty. As yet this course is only being run at Manchester, but the Middlesex Hospital in London is planning to provide a course in the future. A short weekend course for nonmedical workers in STD clinics is run each year by the Sheffield Area Health Authority.

The nurses in the study were asked if they knew of the existence of the six month course. Eighty six (73\%) said they did know of it, but only seven (6\%) nurses in the survey had taken the course, three of these in 1979 and the others in 1975 to 1978. The nurses interviewed were told what training in STD was available, and they were asked if they would like the opportunity to attend the six month or short course if that were possible. Eight nurses (7\%) wanted to attend the ENB longer course and $86(73 \%)$ the short course. Twenty four nurses were not interested in taking any course because either they were near retirement age or they had been in the specialty so long that they felt they could be taught little or nothing. 
TABLE II Views of 69 doctors on suggested further training schemes for nurses

\begin{tabular}{ll}
\hline Type of training & No (\%)* \\
\hline $\begin{array}{l}\text { Practical instruction and assessment in clinic } \\
\text { Training programme incorporating clinic and }\end{array}$ & $27(39 \cdot 1)$ \\
other hospital depts (possibly area scheme) & $10(14 \cdot 5)$ \\
ENB short course (No 932) for nurses & \\
already working in the specialty & $39(56 \cdot 5)$ \\
ENB six month course (No 275) & $18(26 \cdot 1)$ \\
Other & $14(20 \cdot 3)$ \\
\hline
\end{tabular}

*Some doctors thought that more than one scheme was appropriate.

$\mathrm{ENB}=$ English National Board.

\section{DOCTORS' VIEWS ON FURTHER TRAINING FOR} NURSES

The expansion of the role of nurses in the specialty was discussed during the interviews with the doctors, and their views on appropriate further training schemes were sought. To many doctors this was an idea not previously considered, so four suggested types of further training were offered for their consideration (see table II). Of the 69 doctors, 27 (39\%) thought that practical instruction and assessment in the clinic would be useful, and 10 (14\%) favoured a training programme that incorporated other specialties, such as gynaecology, urology, microbiology, and dermatology. Over half the doctors (57\%) were happy to allow and encourage their nurses to attend a short course, but only a quarter (26\%) would have liked them to go on a long course.

\section{JOB SATISFACTION OF NURSES}

GUM has always tended to be a "cinderella" service in terms of nursing and medical recruitment. It was therefore thought to be important to take the opportunity, during the interviews, of asking senior nurses working in the specialty why they had chosen their job, what they found most and least satisfying about it, and if they felt that the role of the nurse in the clinic should change in the future.

TABLE III Reasons of 118 nurses for choosing the specialty

\begin{tabular}{lr}
\hline Reason given & No $(\%)^{*}$ \\
\hline Part of job in outpatients & $34(28 \cdot 8)$ \\
Social hours & $33(28 \cdot 0)$ \\
Something different, change, challenge & $27(22 \cdot 9)$ \\
Interest in the specialty & $17(14 \cdot 4)$ \\
Extra responsibility or promotion & $13(11 \cdot 0)$ \\
Seconded as student and returned & $12(10 \cdot 2)$ \\
$\quad$ when qualified & $5(4 \cdot 2)$ \\
Less physically taxing & $2(1 \cdot 7)$ \\
Liked autonomy & $11(9 \cdot 3)$ \\
Other & $1(0 \cdot 8)$ \\
Not known &
\end{tabular}

TABLE IV No(\%) of 118 nurses specifying satisfying aspects of working in genitourinary clinic

\begin{tabular}{ll}
\hline & No $(\%)^{*}$ \\
\hline Counselling and reassuring patients & $42(35 \cdot 6)$ \\
Contact with patients & $35(29 \cdot 7)$ \\
Extended role of the nurse & $28(23 \cdot 7)$ \\
All aspects of work & $8(6 \cdot 8)$ \\
Teaching patients & $7(5 \cdot 9)$ \\
Teaching nurses & $6(5 \cdot 1)$ \\
Variety of work & $5(4 \cdot 2)$ \\
Not known or other & $9(7 \cdot 6)$ \\
None & $2(1 \cdot 7)$ \\
\hline
\end{tabular}

*93 nurses gave one, 14 gave two, and eight gave three aspects.

Reasons for choosing to nurse in department

Most (29\%) nurses had not chosen the subject specifically, but it was just one of the outpatient clinics to which they were allocated (table III). Almost the same proportion $(28 \%)$ had chosen the specialty because of the convenient working hours. The third most popular reason for working in GUM was that it was something different, a change from other nursing jobs and therefore a challenge (23\%). Other similar reasons were that it was an interesting subject (14\%) and offered extra responsibility and sometimes promotion (11\%). Twelve male nurses who had been seconded to a clinic during their student training had found this period stimulating enough to return after qualifying.

\section{Satisfactory aspects of the job}

Not unexpectedly, for most nurses contact with patients had priority (table IV). Forty two nurses (36\%) were specific in saying that reassuring and counselling patients was most important for them, almost as many $(35,30 \%)$ said just "patient contact" or "dealing with patients", and $28(24 \%)$ found the extended role of the nurse (examining patients and performing microscopy and venepuncture) most satisfying. These are skills that not only. stretch their knowledge and ability but also give more opportunity for nurses' customary physical and verbal contact with patients. Teaching came fairly low on the list of job satisfaction. Only six (5\%) mentioned teaching nurses and seven $(6 \%)$ teaching patients.

\section{Unsatisfactory aspects of job}

It was encouraging that most $(39,33 \%)$ replies in this section said that no area was unsatisfactory (table V). In the smaller part time clinics where the clerical and administrative work was carried out by nursing staff, 27 (23\%) of the nurses found that this was time consuming and detracted from their clinical role. Twelve (10\%) found their work repetitive, usually in clinics where the nurse's role was limited to chaperoning and preparing patients for examination. An equal number 
TABLE V No (\%) of 118 nurses specifying unsatisfactory aspects of working in genitourinary clinic

\begin{tabular}{lc}
\hline & No $(\%)$ \\
\hline None & $39(33 \cdot 1)$ \\
Clerical and administration work & $27(22 \cdot 9)$ \\
Others & $15(12 \cdot 7)$ \\
Lack of appreciation by patients & $12(10 \cdot 2)$ \\
Repetitive work & $12(10 \cdot 2)$ \\
Interpersonal relationships & $5(4 \cdot 2)$ \\
Treating genital warts & $4(3 \cdot 4)$ \\
Lack of teaching & $3(2 \cdot 5)$ \\
All & $3(2 \cdot 5)$ \\
Lack of promotional structure & $1(0 \cdot 8)$ \\
Not known & $3(2 \cdot 5)$ \\
\hline
\end{tabular}

found it difficult to adjust to patients' lack of appreciation for the STD service, which diminished their own role.

According to five (4\%) of the sample, interpersonal relationships were often strained, which greatly detracted from job satisfaction. When doctors travelled to several clinics there was little time to develop continuity of teaching and professional relationships with nurses, who themselves were often moved from the department of GUM to other outpatient departments. Only one nurse said that lack of promotion was the least satisfying aspect of the job, though many nurses mentioned the lack of career structure. Fifteen nurses gave other single reasons, such as poor working facilities, staff shortages, lack of responsibility, and the fact that the interesting patients were referred to larger clinics.

\section{WHAT IS POTENTIAL ROLE FOR NURSES?}

The final aspect covered during the interviews with the nurses was how their jobs could be changed or expanded. As many as 25 nurses (21\%) felt happy with the status quo (table VI). Most thought that changes should only take place after discussion and

TABLE VI Ways that 118 senior nurses thought their role in STD clinics could be expanded

\begin{tabular}{ll}
\hline Extra duties suggested & No (\%) \\
\hline Microscopy & $43(36 \cdot 4)$ \\
None & $25(21 \cdot 2)$ \\
Follow up tests (female patients) & $24(20 \cdot 3)$ \\
Counselling & $20(16 \cdot 9)$ \\
Follow up history and examination (both & $16(13 \cdot 6)$ \\
sexes) & $13(11 \cdot 0)$ \\
New patient examination & $10(8 \cdot 5)$ \\
New patient history & $8(6 \cdot 8)$ \\
Follow up tests (male patients) & $8(6 \cdot 8)$ \\
Prescribing routine drugs & $9(7 \cdot 6)$ \\
Teaching & $4(3 \cdot 4)$ \\
$\quad$ In community and schools & $2(1 \cdot 7)$ \\
Nurses &
\end{tabular}

instruction, and they had no wish to usurp the doctor, only to give the patient a better service and make the best use of their own potential. In some cases changing the nurse's role would necessitate reorganising the clinic procedure and this would cause changes in the roles of other members of the team. Most nurses thought that such modifications would not take place.

Twenty (17\%) of the nurses thought they were appropriate and able to counsel patients. Contact tracing in smaller clinics could also be accomplished by nurses, though this was the opinion of only two (2\%). Teaching in the community, particularly in schools, was mentioned by nine nurses $(8 \%)$. They thought that an approachable and well informed nurse could do much to dispel the fear and stigma attached to STDs in the community, while giving positive guidance. Educating nurses was suggested by four (3\%) of those interviewed as a method of increasing the knowledge of student nurses and encouraging recruitment, and these nurses thought that this was well within the senior clinic nurses's ability.

Most suggestions, however, were to extend the nurse's role in the clinical field. As shown previously, some tasks traditionally performed by doctors are already delegated to some of the nurses. ${ }^{1}$ The nurses felt that, with training and some supervision, they would be able to expand their role and provide a more efficient service for patients and doctors, while increasing their own job satisfaction. For some nurses this included taking histories from and examining both new and old patients.

Nurses who listed prescribing drugs as a possible aspect of their work also mentioned that the drugs generally given in this specialty are limited, not covered by the Dangerous Drug Act, and given routinely according to clinic policy. Except groups such as midwives, nurses are not at present authorised by the General Nursing Council to prescribe drugs.

VIEW OF DOCTORS OF FUTURE ROLES OF NURSES, AND THEIR RECOMMENDATIONS FOR RECRUITMENT

It would be unrealistic to consider the nurses' views of their future role in GUM without also consulting the doctors working in the specialty. The final section of the doctors' interview was therefore devoted to asking their opinions about ways in which the nurse's role could be expanded, any difficulties that had been encountered when recruiting nursing staff, and recommendations for improving recruitment.

Of the 69 doctors interviewed, 61 (88\%) said that if nurses were given appropriate training their role in the specialty could be expanded. Individual doctors indicated up to six ways in which the job might expand, and responses covered every aspect of clinic work (table VII). The doctors were given no prompting, and their replies showed similar priorities to those of the 
TABLE VII Suggestions of 69 doctors for expanding role of nurses

\begin{tabular}{lr}
\hline Extra duties suggested & No (\%) \\
\hline Follow up tests (female patients) & $33(47 \cdot 8)$ \\
Staining slides and microscopy & $29(42 \cdot 0)$ \\
Follow up tests (male patients) & $28(40 \cdot 6)$ \\
Tests from all new patients & $14(20 \cdot 3)$ \\
Tests from all patients & $8(11 \cdot 6)$ \\
Tests only when doctor present in clinic & $8(11 \cdot 6)$ \\
Teaching in the community & $7(10 \cdot 1)$ \\
History taking from all new patients & $11(15 \cdot 9)$ \\
History taking from all follow up patients & $12(17 \cdot 4)$ \\
Counselling and teaching patients & $7(10 \cdot 1)$ \\
Prescribing treatment & $7(10 \cdot 1)$ \\
Giving local treatments and performing & \\
pregnancy tests & $3(4 \cdot 3)$ \\
Performing cytology & $3(4 \cdot 3)$ \\
Tests only when doctor absent from clinic & $2(2 \cdot 9)$ \\
Teaching nurses & $2(2 \cdot 9)$ \\
Clerical and administrative responsibility & $2(2 \cdot 9)$ \\
Contact tracing & $2(2 \cdot 9)$ \\
\hline
\end{tabular}

nurses. The general view was that with training and suitable supervision nurses could take routine tests, especially from patients, both male and female, returning for follow up after treatment. Doctors also felt confident to leave Gram staining of slides and diagnosis by microscopy to nurses. Prescribing treatment was again mentioned, doctors stressing that routine treatments and drugs could be given according to clinic policy. Eight doctors did not feel that the nurse's role could be expanded even after further training.

The doctors interviewed were asked whether they had problems recruiting the right quality of nursing staff. Many doctors were not consulted about the choice of nursing staff, but more than half $(52 \%)$ said that they did have problems in recruitment. Where the clinic was carried out on a part time basis and nurses allocated to STD for a few hours from their other outpatient commitments, the doctor often felt fortunate to have any nursing help. In these circumstances the nurse's attitudes were not always suitable for the specialty, and help was given grudgingly. When staff were employed specifically to work in the STD clinic, however, doctors complained that they still were not consulted about staff appointments. This occurs largely because nurses are employed and paid from the nursing budget, and medical staff, though generally consulted on senior appointments, are not consulted when junior staff are employed.

Doctors who had encountered difficulty in recruitment suggested ways of overcoming this, which included improving career structure and working conditions and educating students and senior nursing staff. Financial incentive by offering higher salaries to STD nurses was also mentioned. To some extent this already occurs as nurses in the specialty receive an extra $4 \%$ of their basic annual salary. Some health authorities, however, will only give this payment to nurses working full time in STD, which possibly excludes 119 clinic nurses in England and Wales.

\section{Discussion}

Doctors and nursing staff in the study recognised that good teaching of student and pupil nurses during their training may gradually remove the stigma and myths about STD, and improve recruitment of nurses to the specialty.

Only $74 \%$ of the doctors interviewed taught student and pupil nurses, and many had very limited time in which to cover the subject. Lack of clinic facilities or space often made it impossible for students to work or observe in clinics during their training. Most (90\%) of the doctors taught the nurses working in their clinic. Teaching was informal and usually included occasional discussion of a patient's management. This type of teaching was offered once practical skills necessary for the running of the clinic had been mastered.

Nearly all (97\%) the nurses interviewed had been given some training, though this was often only just sufficient for them to practice as a useful member of the clinic staff. Theoretical teaching was usually related to a particular patient, and given in a few spare moments snatched between patients. Time was always limited, especially where doctors were peripatetic.

Almost half (42\%) the nurses interviewed felt that the teaching they had been given in the clinic was inadequate, and the opportunities for self education for these nurses were insufficient. Most nurses (91\%) wanted to improve their knowledge by taking a short course. Because of the lack of time, facilities, and (sometimes) adequate pathology facilities in the clinic, we recommend formal training schemes for nurses in the specialty. Two pratical problems would, however, be associated with such schemes. Firstly, nurses working in part time clinics cannot easily be released, especially when a clinic has only one nurse, and adequate cover needs to be arranged; secondly, centres would need to be identified and resources found for teaching.

Despite these problems, a uniform basic training and identification with other nurses in the specialty would clearly improve morale and recruitment. The six month course (no 275) recognised by the England National Board is good training for nurses who are new to the specialty, and is certainly already improving standards of nursing in STD, but secondment for six months for nurses already in post is not always practical. In view of this, we suggest that a series of short courses covering basic practical skills, such as Gram staining and microscopy and an introduction to the diseases, might be held nationally. These courses would also be useful as a forum for discussing further 
training, improving communicatioins and cross fertilisation, and removing some of the isolation felt by nursing staff. These basic courses could subsequently be supplemented by updating sessions.

No particular factors motivated nurses to work in the specialty, though once on the staff of a clinic they enjoyed their work. This highlights the importance of teaching the STDs to nurses in training. These better informed nurses would, we hope, be stimulated to work in clinics for reasons other than that they provide socially convenient hours. The aspects of the job that were most enjoyed might profitably be used to attract more nurses into STD. The reaction of many nurses when first asked about the most satisfying aspects of their role was that they could not identify any particular area but enjoyed coming to work, which is surely a good recommendation.

One of the reasons for nurses entering the profession is a need to "help people", the contact with patients therefore being an essential component of a nurse's job satisfaction. Nurses in the specialty mentioned the attraction of the variety in the work and the differences between this and many other nursing jobs. To retain the interest of nurses working in STD, however, and to improve recruitment, ways of expanding their role should be considered. Both doctors and nurses felt that with training and supervision nurses could expand and extend their role. Teaching and counselling patients and teaching other learners was seen as an obvious expansion of the nurse's role in the specialty. Taking routine tests was generally felt to be appropriate and would provide greater interest and better job satisfaction.

Little interest was thought to be taken in recruiting suitable nurses for the specialty. One suggestion is that each region has a senior nurse to recruit and administe nursing staff for several small clinics and provid, regular in service training. Some areas (East Yorkshir and North Wales) have a peripatetic team of a consultant and nursing staff, which serves several clinics in an area and provides coordinated care. There is certainly a place for a clinical nurse specialist along American lines. The advanced knowledge of skills possessed by some nurses receive no formal recognition, as there are no established posts that would identify and encourage the enlargement of the exceptional contribution that nurses can make.

The incidence of STDs is increasing steadily. New conditions are evolving, and greater public awareness is leading to greater use of an already fully stretched service. Nurses obviously play an important part in this service, and if clinics are to make the most of their potential three areas should be given special attention: (1) student nurses need more teaching about STD, both to increase their knowledge and understanding of diseases and patients, and to make them aware of this specialised area of nursing after they qualify; (2) training programmes aimed at preparing nurses for their specialised role in clinics, and updating their knowledge on a regular basis, should be instituted; and (3) once they are in post, nurses should have their interest maintained by expanding their role to encompass some patient examinations, microscopy, and counselling, and by offering a career structure that reaches beyond sister or charge nurse level.

Nurses are vital members of the team in a department of GUM clinic. With imagination it should be possible to improve their teaching, recruitment, and role, with obvious benefits for the whole of the STD service.

\section{Reference}

1. Rogers JS, Adler MW. Role and training of nurses working in departments of genitourinary medicine in England and $\mathrm{W}$ ales. 1. Role. Genitourin Med 1987;63:122-6. 